\title{
ONTOLOGY-BASED HUMAN-MACHINE COLLECTIVE INTELLIGENCE ENVIRONMENT FOR DECISION SUPPORT
}

\author{
Alexander Smirnov and Andrew Ponomarev \\ St. Petersburg Federal Research Center of the Russian Academy of Sciences (SPC RAS) \\ Russian Federation, 199178, St. Petersburg, $14^{\text {th }}$ Line, 39
}

\begin{abstract}
The paper proposes the design of a computer environment aiming at the support of human-machine collective intelligence in decision-making problems. The environment supports two main processes taking place during the collective effort on a complex problem: process organization (planning the activities and the distribution of the responsibilities) and building a solution (plan implementation). To address the problem of human-machine interoperability, the environment leverages multi-aspect ontologies, allowing to represent the status of the process and information about the problem in a human- and machine-readable way. The proposed solution can be employed in building interoperable human-machine collaborative systems in variety of complex problem domains.
\end{abstract}

\section{KEYWORDS}

Human-Machine Systems, Human-In-The-Loop, Collective Intelligence, Collaboration, Decision Support

\section{INTRODUCTION}

Many real-life problems are too complex to be addressed by purely computer/software systems (even despite new promising developments in the area of $\mathrm{AI}$ ). This is especially true, for example, for the problems arising in managing complex business or societal systems. Besides, decision-making is usually connected with some form of responsibility and accountability, but the ideas and technologies for algorithmic accountability, though being actively explored, are still not mature enough for practical uses. Therefore, human experts still are (and probably for a rather long time will be) an important part of many complex decision-making scenarios. However, human experts today usually are accompanied by the increasing use of AI tools and more and more sophisticated software agents.

In this paper, the problem of supporting human-machine collaboration in decision-making scenarios is explored in the following perspective. As the global communication technologies evolved, a new approach to building information processing applications has emerged, offered by crowdsourcing technology. Today, one can reach a huge (global) pool of potential experts and contributors, possessing all the skills and knowledge required for solving a particular problem. However, crowdsourcing is typically used for rather simple tasks (like, image or audio annotation), the possibility of using it for more complex problems is the subject of active research (Kittur et al., 2011; Retelny, Bernstein and Valentine, 2017; Valentine et al., 2017). Besides, in the vast majority of crowdsourcing applications, the participants are not able to communicate (the tasks issued to them, are so simple that communication and collaborative work are not usually reasonable).

This paper focuses on supporting the collective intelligence of dynamic ( $a d$ hoc) teams consisting of heterogeneous (human and machine) participants. In a sense, this research is positioned between classical group decision support systems (taking from them protocols for collective decision-making and agreement), modern crowdsourcing applications (taking from them the techniques addressing workforce on the on-demand basis), and multi-agent human-machine collaboration. Besides, the ad hoc teams (not necessarily in the Internet) are an interesting phenomenon, actively studied from the organization research point of view (Bechky, 2006; Faraj and Xiao, 2006). 
The paper describes the conceptual organization of the human-machine collective intelligence environment, as well as some aspects of technical implementation. It also illustrates how different parts of the environment interact during the collective decision support scenarios.

The rest of the paper is structured as follows. Section 2 describes related research in a several areas influencing the concept and design of the environment. Section 3 describes the main ideas behind the proposed environment design and its mechanisms.

\section{RELATED WORK}

The problem of building environments allowing to access the resources of loosely connected people interacting via Internet is often discussed as one of the lines in crowdsourcing or crowd computing research. Despite the fact, that in most of the crowdsourcing platforms participants usually do their tasks independently, in crowdsourcing research there are continuous attempts being taken to adapt crowdsourcing to more complex problems and (these trend are related) to develop collaborative workflows (Retelny et al., 2014). It has also been shown, that for complex problems pre-programmed workflows (typical for most of the crowdsourcing platforms) are too limited (e.g., (Retelny, Bernstein and Valentine, 2017)), therefore, the potential of crowdsourcing for complex work is tightly connected with the mechanisms of dynamic team formation, workflow adaptation. In particular, the papers (Retelny et al., 2014; Retelny, Bernstein and Valentine, 2017; Valentine et al., 2017) study the limitations of workflow-based solutions, and propose ways to overcome these limitations with a help of dynamic organizations from members of the crowd (one of them is called "flash organizations" (Valentine et al., 2017)). However, these papers address only problems of human collectives. In this research, however, we are building an environment where heterogeneous agents (human and software) would be able to collectively decide on the details of the workflow.

Collaborative decision-making is also actively explored in the area of computer-supported collaborative work (e.g., (Terveen, 1995; Elmarzouqi, Garcia and Lapayre, 2008)). The results of the Dicode project implemented within the framework of the European FP7-ICT program (Karacapilidis, 2014; Karacapilidis and Tampakas, 2019) deserve special attention. In the framework of the project, in particular, an ontological presentation of the argumentation process and a number of visual tools for working with a thus formalized set of interrelated arguments are proposed. The importance of these results lies in the fact that for sharing information about the problem by experts and software agents, it should have a structured representation, one of the options of which is a graph of the relationship of arguments. However, explicitly encoding all the arguments may be too difficult.

A high-level paper (Jennings et al., 2014) summarizes what is required to support human-machine collectives and current technological limitations for building them. In particular, the authors underline the need for the flexible autonomy of the agents, agile team-building, incentivization and accountability. The proposed environment account for all these prerequisites.

In a broader perspective, an important source line of research relevant for such an environment is the study of ad hoc teaming and collaboration in human collectives. Dynamic creation of (relatively) short-lived teams is typical in some areas of human activity. The findings of socio-psychological research of mechanisms allowing efficient composing and functioning of such teams can be useful for any computer-based environment supporting such teams (although, not all of them are directly applicable).

There are several important publications in this area. Faraj and Xiao (2006) analyze the coordination processes of medical trauma center where fast-response and error-free activities are essential requirements. They find two categories of coordination mechanisms employed in such organizations: expertise coordination practices (used most of the time, on the habitual patient's trajectory - reliance on protocols, plug-and-play teaming, community of practice structuring, knowledge sharing), and dialogic coordination practices (used for a problematic patient's trajectory - epistemic contestation, joint sense-making, cross-boundary intervention, protocol breaking). Bechky (2006) analyzes and describes coordination mechanisms of a filming crew (filming crews are usually collected for relatively short period of time from people most of whom previously didn't work together). Both papers underline flexible role-based distribution of responsibilities as a key to coordination in ad hoc teams.

Overall, there are following outcomes that can be derived from the analysis of the organization research, that may solve as requirements for the environment: 
1) Research of social systems and current practices in designing such systems agree that the elements of self-organization are critical, especially when solving complex problems. Despite the fact that there are a number of works close to the proposed, there are currently no solutions supporting the collective intelligence formed by artificial intelligent software services and people.

2) An important mechanism for self-organization in dynamic human groups is role-based coordination (Retelny, Bernstein and Valentine, 2017; Valentine et al., 2017). Non-rigid role responsibilities structure has shown to be very effective in supporting collaboration in ad hoc teams. The concept of the role, therefore, should also be provided by the environment, as well as the mechanism of "switching" the software agent between the roles (if possible) and clarifying the specific content of the role within the framework of this team (Lhaksmana, Murakami and Ishida, 2018).

3) The manifestation of team self-organization can be structured using patterns (Gilbert et al., 2015), structures and coordination schemes that participants come to under certain conditions.

4) A formal representation of problem-related information is necessary, allowing one to track the cause of certain arguments or results (provenance) (Jennings et al., 2014; Karacapilidis and Tampakas, 2019), which is "understood" by both the participating people and the software agents. Therefore, information exchange infrastructure should not only allow all participants to get relevant information, but also to support accountability and provenance.

5) The environment should provide some incentive management mechanism allowing to reward participants (Jennings et al., 2014). It is crucial due to the openness of the environment.

\section{COLLABORATION ENVIRONMENT DESIGN}

The purpose of the environment is to leverage the coordination procedures between heterogeneous agents (in short-lived teams), allowing information exchange on two levels: information concerning the problem being solved (available data, opinions, arguments and models), and process information (role distribution, responsibilities and so on).

It should be noted, that the environment is inherently dedicated to decision support problems. Therefore, the design is influenced by decision-making methodologies (e.g., (Simon, 1979; Mann, Harmoni and Power, 1991; Guo, 2008)) and the workflow implemented by a team mostly corresponds to a typical decision-making process.

The proposed environment is discussed in this paper in two levels. First, conceptual design, which shows important concepts of the environment and how they interact during the problem-solving. Second, technological design, showing how (by what technologies and software components conceptual design is implemented).

The environment interacts with the following principal actors: end-user (decision-maker), participant, and service provider. End-user (decision-maker) uses the environment to get help in making a decision. He/she describes the problem and posts so that the problem description is visible to a specified community. Participant is an active entity (human or a software service) working on a problem given by the end-user. Finally, service provider develops, integrates to the environment, and supports software services that can act as participants working on some problem given by the end user. Service provider is also responsible for the deployed services, assuaging the problem of service accountability.

The core entities involved in most of the processes taking place in the environment are problem and team. Problem is introduced by an end-user and then is addressed by a team of participants. The description of a problem has a complex structure and representation. First of all, it contains not only information, specified by the end-user (initial statement), but also includes all the information produced by the team. So, during team's activity, the problem becomes more and more detailed. Second, to enable (at least, partly) effective interpretation by software agents, problem description is represented in a semi-structured way. In particular, machine readability is achieved via using ontologies. It is a crucial point in design. Since their introduction to the IT industry during Semantic Web initiative, ontologies proved themselves both quite efficient in solving interoperability problems and quite non-friendly for non-expert users. Still, we argue that for mixed human-machine teams, the ontologies provide a kind of lingua franca, acceptable by both human and software participants of the process. However, to make the use of ontologies easier for people, the environment makes the use of ontologies as implicit as possible by relying on three techniques: 
- Implicit ontological representation of the structure of problem information. It means, that a human participant sees the problem description as a set of constraints, alternatives, criteria, alternative evaluations, arguments and so on, in other words, in terms that are widely used in decision-making literature and, in particular, by nearly every decision-making methodology. However, "under the hood" this structure is encoded with the decision-making ontology, therefore, software agents are also able to analyze the problem structure.

- Natural language processing. Using advances in this area it is possible to infer the role of some information pieces, its relationship with the goal and/or some line of argumentation and so on.

- GUI-based nudging participants to encode problem structure in an ontology-compatible way. Digital nudging (Schneider, Weinmann and vom Brocke, 2018) is a technique of growing importance, due to the fact that currently so many choices people do by interacting with virtual environments. In particular, nudging is quite effective alongside with the natural language processing of problem information, as it allows to ask user to disambiguate some terms and relations in the problem information.

The environment defines two basic ontologies, representing different aspects of the collaborative decision support:

- Decision-making ontology. This ontology defines main concepts that are used during decision-making (criterion, alternative, evaluation etc.) and interaction between them. The ontology is based on the analysis of existing decision-making methodologies and has been built in such a way to support majority of them.

- Collaboration and coordination ontology. It defines the concepts used in distributing work among team members (role, responsibility, dependency etc.).

The use of these ontologies allow artificial agents to 'understand' the processes taking place in the team and contribute to them. However, for the ontology-based decision-support agents, there is also a possibility to define a domain ontology and map it to the decision-making ontology. These ontologies (two generic and the domain one) capture orthogonal views on the decision-making process. To tie these views into a complex, consistent structure, we propose to use the apparatus of multi-aspect ontologies (Smirnov, Shilov and Parfenov, 2019). It allows connecting multiple conceptualizations (possibly, built using different formalisms); so that changes performed in one view (aspect) are automatically reflected in other views (aspects). For example, if the environment is used in smart tourism applications and the decision to be made is choosing a tourist route, then route (defined in the problem domain ontology) is equivalent to the alternative (defined in the decision-making ontology). The presence of these mappings allows the software participants of the decision support process to treat routes as alternatives (e.g., in visualizing them or building Pareto optimal set (Luc, 2008)). It also allows problem-specific services to contribute to the decision-making (e.g., estimate typical traffic of the route, its length). Obviously, these mappings between different aspects, joining aspect ontologies into a multi-aspect ontology are problem-specific and building these mappings is a part of problem definition.

The way problem information becomes richer and grows via interaction of agents, is implemented via 'blackboard' interactions. Particularly, with a help of ontology-based smart space technology (Roffia et al., 2018). As the progress of a team on the problem is reflected by structural changes of ontological description of the problem, it allows to leverage ontology-based publish-subscribe mechanisms to intelligently perform some actions by software agents in response to particular situations during problem-solving.

Another core entity, as it was already mentioned, is a team. Team in the context of the environment is defined as a heterogeneous group (consisting of human participants and software services) working towards solution of a particular problem. Each problem has a team dedicated to it. Obviously, a participant may be a member of several teams, or not be a member of any team.

Initial team formation is based on the same principles that are used in most of the crowdsourcing platforms and knowledge networks (e.g., (Ahmad et al., 2011)): each participant has a profile describing key specializations, problem-solving history, as well as the history of previous collaborations (with mutual evaluations). The initiative in this process is mixed in the sense that a contributor should send a proposal to the end-user, consisting of one or more team members (proposal may include several participants that already have some positive experience of working together), and end-user has to collect the initial team. However, decisions of the both parties - participants and end-users - are assisted by environment. The participants may choose to receive recommendations in case some problem touching his/her area of competences is posted. On 
the other hand, end-users may explore the description and history of all the participants mentioned in the proposals. If a team understands that it lacks some competencies or resources, it may create a new resource requirements, that are registered in the environment and resolved in a manner, similar to the initial team formation process (participants have to actively apply for the positions in the team, however, both sides are assisted by the environment mechanisms).

This doesn't fully apply to the software participants (services). As the throughput of software services is not as limited as the throughput of humans, and the execution is relatively cheap, software services are passively connected to any team and by the mechanisms of the environment (ontology-based publish-subscribe) are watching the processes taking place with the problem. There are two states a software service can be in with respect to a team (Figure 1): dormant and active. Initially, all services are in the dormant state and are waiting specific conditions during the problem-solving. If these conditions defined by a particular service are met, the service tries to activate, describing its purpose and terms of use. If the team agrees that the service is useful for the problem, the service is allowed to activate (change state to active) and become a member of the team. Otherwise, the service remains dormant. Active service may also be transferred to the dormant state by a decision of the team. Besides, the services can be accessed via a service catalog and activated manually by team members.

Active services can be used by the members of a team. The mechanics of their usage depends on the kind of a service. There are following types of services:

- Problem-solving service;

- External tool and database access service.

Problem-solving service accesses the problem information described in the form of ontology and natural text and can actively add information pieces to it. An example of such service is a statistics-based question answering service - if it detects a question about some facts (e.g., "How many people die from tuberculosis in the World in one year?") and can answer it in some form it adds an answer to the question. Another example is a service that derives from the problem information a current set of alternatives and their evaluations, builds a Pareto optimal set and adds it to the problem information.

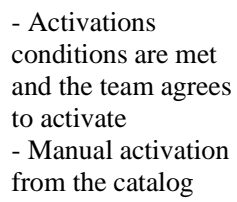

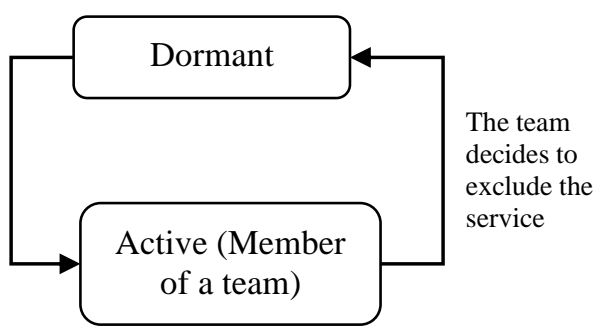

Figure 1. Service states with respect to a team

External tool and database access services in activated form only provide an access to a specified resource. For example, if a team need epidemic database, it can activate the service that grants access to this database and use it for queries.

Simultaneously two processes take place when team works on a problem: solution preparation and decision support (re)organization. Both of these processes are supported by mechanisms provided by the environment. Solution preparation is main productive process, during which problem is enriched with new information and artifacts created by team members. General scheme of the solution preparation process is driven by decision support methodologies (e.g., Simon's model (Simon, 1979), DECIDE (Guo, 2008), or GOFER (Mann, Harmoni and Power, 1991)). It should also be noted, that these methodologies provide the general scheme of the solution preparation, main stages that have to be performed, while execution of each stage is done via activities relevant to the problem at hand. For example if the environment is used by an organization to decide which motivation policy to implement for the workers, then the stage of Considering all the alternatives may contain a task of identifying relevant scientific publications about the effectiveness of motivation policies. The necessary activities (contents of the respective stage) are defined and enacted by the team itself, the environment allows to track these activities and connect their results with the problem definition and the concepts of decision-making ontology. 


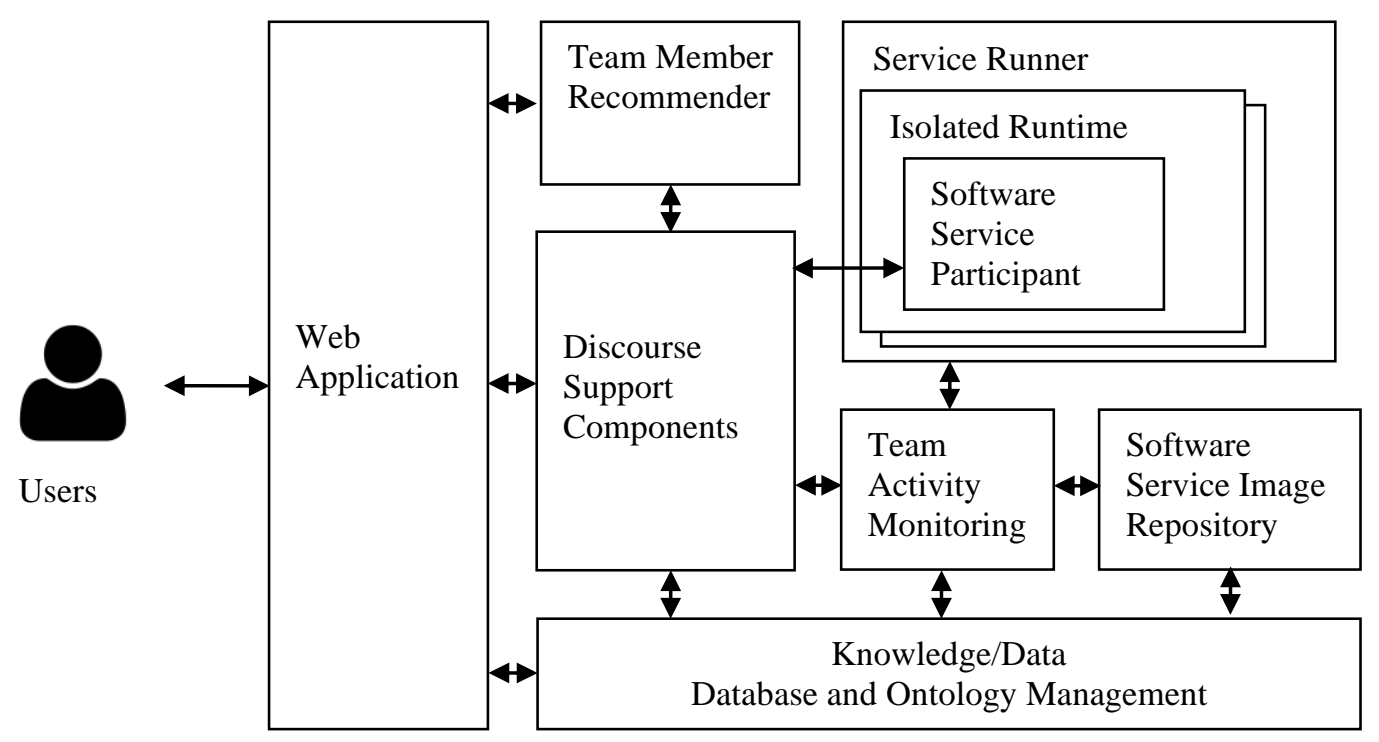

Figure 2. Technological design of the environment

Physically, the process of solution preparation can be viewed as adding information to the problem definition initially provided by the end-user (in the smart space). First, by adding explicit criteria and constraints (during the activities of initial stages - Define the problem and Establish the criteria), then by adding alternatives and their evaluations (later stages). The result of this process is fully detailed description of a problem situation, weighted alternatives and their estimated consequences - accepted by the end-user.

Decision support (re)organization process represents all the activities aimed on planning and organization of team work (e.g., deciding whether additional resources are required, assigning team member responsibilities, setting task deadlines and identifying new tasks to be solved in order to reach the goals of the whole process). Complex activities cannot be fully planned (and the literature review supports that), therefore, the team can adapt the plan as the solution process advances. General scheme of the decision support (re)organization process includes a number of activities: plan, refine, execute, observe, and reflect. During the Plan activity the team (or a responsible member of the team) builds a list of activities and assigns responsibilities using the features of the environment. If it is necessary, some plan items should be refined (this is especially the case when there are software services assigned to them). The planned activities are executed (this is actually a solution preparation process, described earlier); the results are observed and reflected on. If during the execution it turns out that some additional activities have to be performed, then the plan is adjusted and the cycle continues.

Technologically the environment (Figure 2) is implemented as a web application where each problem (and, therefore, a team) has its own workspace, supporting both informal text-based communication (similar to popular collaboration environments like Slack (Slack - Official Site, no date) or Mattermost (Mattermost - Official Site, no date)), but also have integrated 'nudging' modules allowing to tie the information into decision-making ontology formalized view, making it accessible for software services. Besides, humans can also browse the problem information in this structured ontology-based view and edit it if it is necessary.

The 'nudging' functionality is implemented as a set of Discourse Support Components, that maintain both the structured (ontology-based) and unstructured problem description and constantly perform mapping between them. Besides, these components provide an interface for other components of the system to access the contents and requirements of the team.

Team Member Recommender is responsible for performing assistance on finding team members for problems. It issues recommendations to participants who fit the active teams according to the implemented fit estimation models.

Software services are integrated into the collective intelligence environment in the following way. Service provider registers the service by specifying activation context, terms of usage and runnable image of a 
service (Software Service Image Repository component). The description is encoded via SPARQL-based declarative language to enable ontology-based subscription mechanisms. In order to preserve confidentiality of the team work, the services are run in an isolated way. Until their presence is accepted by the team, watching is done by the environment (on behalf of a service, particularly, by Team Activity Monitoring component). After the activation condition has been fired, a service instance is created from the runnable image and run in a new isolated container without internet access making sure that details of the problem will not be accessible by service provider who is not member of a team.

\section{CONCLUSION}

The paper addresses some conceptual and technological issues arising in creating dynamic collaborative systems for complex problems decision-support. Foremost, it addresses the issue of interoperability in heterogeneous (human and machine) collectives working on a decision support problem, and the issue of adapting the workflow (action plan) of a complex collaborative activity, which is important, as reasonably complex decision-support procedures cannot be designed in advance in the sufficient level of detail.

Based on the multi-disciplinary analysis of team coordination and collaborative systems research, the paper proposes a design of a novel class of environments, providing means for human-machine collective intelligence for decision support.

The issue of interoperability is addressed with the help of ontologies providing human- and machine-readable representation of the both problem- and process-related information. In particular, the environment leverages the idea of multi-aspect ontologies, allowing to connect problem domain and universal concepts of decision-making and team functioning in a problem-specific way.

Technologically, the environment derives many ideas from ontology-based smart space technology, providing a semantic shared space, readable and modifiable by team members. Smart space technology also serves as an interaction media, implementing indirect communication via publish/subscribe mechanism. In particular, this mechanism allows software team members to react to certain structures in semantic representation of the decision-making problem.

The proposed environment can be used to support decision-making in a variety of domains, where inherent complexity of the problems requires the symbiosis of human's creative potential, machine's information processing ability, and the flexibility in organizing the interactions between them.

\section{ACKNOWLEDGEMENT}

The research is funded by the Russian Science Foundation (project \# 19-11-00126).

\section{REFERENCES}

Ahmad, S. et al. (2011) 'The jabberwocky programming environment for structured social computing', Proceedings of the 24th annual ACM symposium on User interface software and technology - UIST '11. New York, New York, USA: ACM Press, pp. 53-64. doi: 10.1145/2047196.2047203.

Bechky, B. A. (2006) 'Gaffers, gofers, and grips: Role-based coordination in temporary organizations', Organization Science, 17(1), pp. 3-21. doi: 10.1287/orsc.1050.0149.

Elmarzouqi, N., Garcia, E. and Lapayre, J.-C. (2008) 'CSCW from Coordination to Collaboration', in, pp. 87-98. doi: 10.1007/978-3-540-92719-8_9.

Faraj, S. and Xiao, Y. (2006) 'Coordination in fast-response organizations', Management Science, 52(8), pp. 1155-1169. doi: $10.1287 / \mathrm{mnsc} .1060 .0526$.

Gilbert, N. et al. (2015) 'Self-organizing dynamical systems', International Encyclopedia of the Social \& Behavioral Sciences. Elsevier.

Guo, K. L. (2008) 'DECIDE: a decision-making model for more effective decision making by health care managers', The Health Care Manager, 27(2), pp. 118-127. doi: 10.1097/01.HCM.0000285046.27290.90. 
Jennings, N. R. et al. (2014) 'Human-agent collectives', Communications of the ACM, 57(12), pp. 80-88. doi: $10.1145 / 2629559$.

Karacapilidis, N. (ed.) (2014) Mastering Data-Intensive Collaboration and Decision Making. Cham: Springer International Publishing (Studies in Big Data). doi: 10.1007/978-3-319-02612-1.

Karacapilidis, N. and Tampakas, V. (2019) 'On the Exploitation of Collaborative Argumentation Structures for Inducing Reasoning Behavior', in Proceedings of the 18th International Conference on WWW/Internet 2019. IADIS Press, pp. 78-84. doi: 10.33965/icwi2019_201913L010.

Kittur, A. et al. (2011) 'CrowdForge: Crowdsourcing Complex Work', in Proceedings of the 24th annual ACM symposium on User interface software and technology UIST'11.

Lhaksmana, K. M., Murakami, Y. and Ishida, T. (2018) 'Role-Based Modeling for Designing Agent Behavior in Self-Organizing Multi-Agent Systems', International Journal of Software Engineering and Knowledge Engineering, 28(01), pp. 79-96. doi: 10.1142/S0218194018500043.

Luc, D. T. (2008) 'Pareto Optimality', in Pareto Optimality, Game Theory And Equilibria. Springer Optimization and Its Applications, vol 17. Springer, New York, pp. 481-515. doi: 10.1007/978-0-387-77247-9_18.

Mann, L., Harmoni, R. and Power, C. (1991) 'The GOFER course in decision making', in Baron, J. and Brown, R. V. (eds) Teaching decision making to adolescents. Hillsdale: Lawrence Erlbaum Associates, pp. 61-78.

Mattermost - Official Site (no date). Available at: https://mattermost.com/.

Retelny, D. et al. (2014) 'Expert crowdsourcing with flash teams', Proceedings of the 27th annual ACM symposium on User interface software and technology - UIST '14, pp. 75-85. doi: 10.1145/2642918.2647409.

Retelny, D., Bernstein, M. S. and Valentine, M. A. (2017) 'No Workflow Can Ever Be Enough: How Crowdsourcing Workflows Constrain Complex Work', Proceedings of the ACM on Human-Computer Interaction, 1(2), p. Article 89. doi: $10.1145 / 3134724$.

Roffia, L. et al. (2018) 'Dynamic Linked Data: A SPARQL Event Processing Architecture', Future Internet, 10(4), p. 36. doi: 10.3390/fi10040036.

Schneider, C., Weinmann, M. and vom Brocke, J. (2018) 'Digital nudging', Communications of the ACM, 61(7), pp. 67-73. doi: 10.1145/3213765.

Simon, H. (1979) 'Rational Decision Making in Business Organizations', American Economic Association, 69(4), pp. 493-513.

Slack - Official Site (no date). Available at: https://slack.com/

Smirnov, A., Shilov, N. and Parfenov, V. (2019) 'Building a Multi-aspect Ontology for Semantic Interoperability in PLM', in Product Lifecycle Management in the Digital Twin Era. IFIP Advances in Information and Communication Technology. Springer, pp. 107-115. doi: 10.1007/978-3-030-42250-9_10.

Terveen, L. G. (1995) 'Overview of human-computer collaboration', Knowledge-Based Systems, 8(2-3), pp. 67-81. doi: 10.1016/0950-7051(95)98369-H.

Valentine, M. A. et al. (2017) 'Flash Organizations', in Proceedings of the 2017 CHI Conference on Human Factors in Computing Systems - CHI '17. New York, New York, USA: ACM Press, pp. 3523-3537. doi: $10.1145 / 3025453.3025811$. 